\title{
Predictive Torque and Flux Control of an Induction Machine Fed by a Voltage Source Inverter
}

\author{
Marco Rivera, José Riveros, Consuelo Rodríguez, Patrick Wheeler and Jaime Rohten
}

\begin{abstract}
Model predictive control strategies have recently emerged as a viable alternative for the control of power electronic converters in a range of applications. In this paper a new model predictive control technique for both the ux and torque of an induction machine is presented. The algorithm selects from the available switching states of the voltage source inverter, the chosen state in each sample period optimizes a cost function which is based on the tracking of the electromagnetic torque and the stator ux together with additional criteria to ensure the proper operation of the machine. The results demonstrate the fast torque-ux response with the required quasi sinusoidal stator machine currents.
\end{abstract}

Keywords-Induction machine, model predictive control, predictive torque control, voltage source inverter.

\section{INTRODUCCIÓN}

La estrategias de control directo de torque y flujo han demostrado ser las más eficientes en el control de velocidad de máquinas de inducción en donde uno de los principales requisistos es una más elevada velocidad de respuesta [1], [2], en comparación con el tradicional control vectorial (FOC) [3], [4]. En estos esquemas, las variables de control son el flujo de estator y el torque electromagnético, mientras que también se debe obtener una corriente de estator con un mínimo contenido de armónicas no deseadas [5].

Dentro de la clasificación de controladores descrita, el más tradicional es el control directo de torque (DTC) [6]. Esta técnica fue desarrollada durante la década de los 80 , y ha sido ampliamente promocionada incluso en prototipos comerciales [7]. Su operación se basa en el empleo de controladores de histéresis (de flujo de estator y torque electromagnético) en conjunto con la posición del vector de flujo del estator para seleccionar, en una tabla de búsqueda, el estado de conmutación del inversor fuente de tensión (VSI) que mantenga el par y flujo dentro de la banda de regulación [8]. Esta arquitectura ha reportado mucha simplicidad en su implementación además de robustez, pero a costa de un alto rizado en las corrientes del estator y dificultad al momento de buscar agregar otros criterios en la ley de control [7]. Esta última limitación reduce el potencial de esta técnica para su adopción en otros diseños

Marco Rivera. Director del Laboratorio de Conversión de Energías y Electrónica de Potencia, Universidad de Talca, Curicó, Chile.

José Riveros. Facultad Politécnica, Universidad Nacional de Asunción, Asunción, Paraguay.

Consuelo Rodríguez. Estudiante de Ingeniería Civil Mecatrónica, Facultad de Ingeniería, Universidad de Talca, Curicó, Chile.

Patrick Wheeler. Power Electronics, Machines and Control Group, The University of Nottingham, Nottingham, UK.

Jaime Rohten. Universidad del Bo Bo, Concepcin, Chile.

978-1-6654-0127-2/21/\$31.00 (C) 2021 IEEE de convertidores de potencia (tales como los de la tecnología multifásica o la multinivel que están siendo ampliamente promovidas en la comunidad científica) [9].

Se han propuesto varias modificaciones e implementaciones alternativas para conseguir el control de variables adicionales y reducir el rizado obtenido con el DTC. Sin embargo, fue el desarrollo del control predictivo el que ha proveído una alternativa viable [10]. El esquema es conocido como control predictivo de torque (PTC) [11].

La arquitectura clásica del PTC se basa en el empleo de un controlador proporcional-integral externo que define el torque de referencia en función al error de velocidad, mientras que el flujo de referencia del estator puede mantenerse constante e igual al valor nominal dentro de la región lineal de velocidad. El controlador interno se compone de un modelo predictivo de torque y flujo, una función de costo y una etapa de optimización de ésta. El modelo predictivo se emplea para predecir el comportamiento del torque y el flujo producido por cada estado de conmutación considerado, mientras que en la función de costo se define la ley de control (en este caso los errores de torque y flujo [12]). En la optimización, las respuestas predichas de un número finito de salidas del VSI son evaluadas con la función de costo y se selecciona la alternativa que la minimiza para aplicarla durante el próximo periodo de muestreo.

Entre las bondades reportadas para el PTC, además de la simple inclusión de criterios adicionales de regulación y funciones no lineales, el empleo de este esquema de control ha reportado una notable reducción del rizado de corriente y un leve incremento de la respuesta dinámica en comparación al DTC [13]. No obstante, estas propiedades son obtenidas en detrimento a un incremento en la carga computacional requerida, que actualmente es soslayada con los modernos controladores digitales tales como FPGA y DSP [14].

El desarrollo del PTC sigue siendo un tema de interés en la comunidad científica. Actualmente, se han desarrollado esquemas basados en esta técnica para obtener mejoras en algunas variables de desempleño, como ser el espectro [14]. Además está siendo extendido en otros diseños de convertidores de potencia [9]. En este artículo se presenta la implementación en simulaciones del PTC en un motor de inducción del tipo jaula de ardilla. Este material busca fortalecer la literatura respecto a este tema, para servir como material de consulta y guía a profesionales, académicos y estudiantes del área de ingeniería eléctrica y electrónica. 


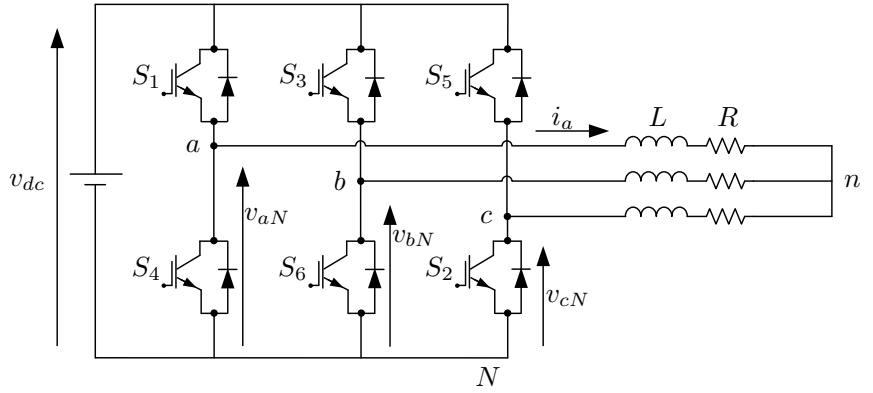

Fig. 1: Circuito inversor fuente de voltaje.

Tabla I: Estados de Conmutación Válidos del VSI

\begin{tabular}{c|cccccc} 
Estado & $S_{1}$ & $S_{2}$ & $S_{3}$ & $S_{4}$ & $S_{5}$ & $S_{6}$ \\
\hline$\# 1$ & 1 & 1 & 0 & 0 & 0 & 1 \\
$\# 2$ & 1 & 1 & 1 & 0 & 0 & 0 \\
$\# 3$ & 0 & 1 & 1 & 1 & 0 & 0 \\
$\# 4$ & 0 & 0 & 1 & 1 & 1 & 0 \\
$\# 5$ & 0 & 0 & 0 & 1 & 1 & 1 \\
$\# 6$ & 1 & 0 & 0 & 0 & 1 & 1 \\
$\# 7$ & 1 & 0 & 1 & 0 & 1 & 0 \\
$\# 8$ & 0 & 1 & 0 & 1 & 0 & 1
\end{tabular}

\section{Modelo Matemático del InVersor Fuente de VOLTAJE}

La función de un inversor de fuente de voltaje (VSI) es cambiar la señal de voltaje continuo de entrada a una señal de voltaje alterno de salida, con una frecuencia y amplitud variables y determinada por el usuario. La topología del inversor fuente de voltaje básicamente viene representado por la Fig. 1, donde es posible ver que este convertidor de potencia consiste de 6 switches unidireccionales.

Para el correcto funcionamiento de este inversor se debe asegurar que los dos interruptores de cada pierna mostradas en la Fig. 1 deben operar de un modo complementario, con el fin de evitar un cortocircuito de la fuente DC. Como resultado, solo ocho posibles estados de conmutación se permiten para este convertidor, con el cual se generan los voltajes de línea a línea y la corriente circulando en el DC-link. El detalle de estos ocho estados válidos de conmutación están indicados en la Tabla I.

La corriente del enlace DC $i_{d c}$ se determina como una función de los switches del inversor y las corrientes de salida i como,

$$
i_{d c}=\left[\begin{array}{lll}
S_{1} & S_{3} & S_{5}
\end{array}\right] \mathbf{i}
$$

El voltaje de salida está sintetizado como una función de los switches del inversor y el voltaje del enlace DC $v_{d c}$, como se muestra en la ecuación (2).

$$
\mathbf{v}=\left[\begin{array}{c}
S_{1} \\
S_{3} \\
S_{5}
\end{array}\right] v_{d c}
$$

\section{Modelo Matemático de la Máquina de INDUCCIÓN}

Las ecuaciones del motor de inducción en un marco de referencia de coordenadas $\alpha \beta$ queda representado por:

$$
\mathrm{v}_{\mathrm{s}}=R_{s} \mathrm{i}_{\mathrm{s}}+d \frac{d \psi_{s}}{d t}+j \omega \psi_{s}
$$

Para la ecuación (4) el voltaje del rotor es igual a 0 , ya que se considera un motor jaula de ardilla.

$$
0=R_{r} \mathrm{i}_{\mathrm{r}}+d \frac{d \psi_{r}}{d t}+j\left(\omega-\omega_{r}\right) \psi_{r}
$$

donde:

- $R_{s}$ y $R_{r}$, corresponden a las resistencias del estator y rotor.

- $\mathrm{i}_{\mathrm{S}} \mathrm{y} \mathrm{v}_{\mathrm{s}}$, son los vectores de corriente y voltaje en el estator respectivamente.

- $\mathrm{i}_{\mathrm{r}}$, es el vector de corriente en el rotor.

- $\psi_{s}, \psi_{r}$, son los vectores de flujo del estator y rotor.

- $\omega$ corresponde a la velocidad de rotación en un marco de referencia arbitrario y $\omega_{r}$, corresponde a la velocidad angular del rotor.

El flujo del estator y rotor de la máquina se definen por:

$$
\begin{aligned}
& \psi_{s}=L_{s} \mathrm{i}_{\mathrm{s}}+L_{m} \mathrm{i}_{\mathrm{r}} \\
& \psi_{r}=L_{m} \mathrm{i}_{\mathrm{s}}+L_{r} \mathrm{i}_{\mathrm{r}}
\end{aligned}
$$

Donde $L_{s}, L_{r}$ y $L_{m}$, corresponden a las inductancias del estator, rotor e inductancia de magnetización.

El torque del motor se define como:

$$
T=\frac{3}{2} p \operatorname{Re} \psi_{s}^{*} \mathrm{i}_{\mathrm{s}}=-\frac{3}{2} p \operatorname{Re} \psi_{r}^{*} \mathrm{i}_{\mathrm{r}}
$$

donde:

- $T$ y $p$, corresponden al torque electromagnético y a la cantidad de pares de polos.

- $\psi^{*}$, es el complejo conjugado de $\psi$.

Las ecuaciones obtenidas se utilizan para estimar el flujo del estator y rotor, además son utilizadas para determinar las predicciones de las seales de corrientes y flujo del estator así como el torque eléctrico, utilizando las ecuaciones en tiempo discreto como se mostrará más adelante en el capítulo 6. Las ecuaciones de la máquina en función de las variables de estado según [15] quedan definidas como:

$$
\begin{gathered}
\mathrm{i}_{\mathrm{s}}+\tau_{\sigma} \frac{d \mathrm{i}_{\mathrm{s}}}{d t}=-j \omega \tau_{o} \mathrm{i}_{\mathrm{s}}+\frac{K_{r}}{R_{\sigma}}\left(\frac{1}{\tau_{r}}-j \omega_{r}\right) \psi_{r}+\frac{\mathrm{v}_{\mathrm{s}}}{R_{\sigma}} \\
\psi_{r}+\tau_{r} \frac{d \tau_{r}}{d t}=-j(\omega-\omega r) \tau_{r} \psi_{r}+L_{m} \mathrm{i}_{\mathrm{s}}
\end{gathered}
$$

donde:

$$
\begin{aligned}
& \text { - } \tau_{r}=\frac{L_{r}}{R_{r}} \\
& \text { - } \tau_{\sigma}=\sigma \frac{L_{s}}{R_{\sigma}} \\
& \text { - } \sigma=1-\frac{L_{m}^{2}}{L_{s} L_{r}} \\
& \text { - } K_{r}=\frac{L_{m}}{L_{r}} \\
& \text { - } K_{s}=\frac{L_{m}}{L_{s}} \\
& \text { - } R_{\sigma}=R_{s}+R_{k} K_{R}^{2}
\end{aligned}
$$




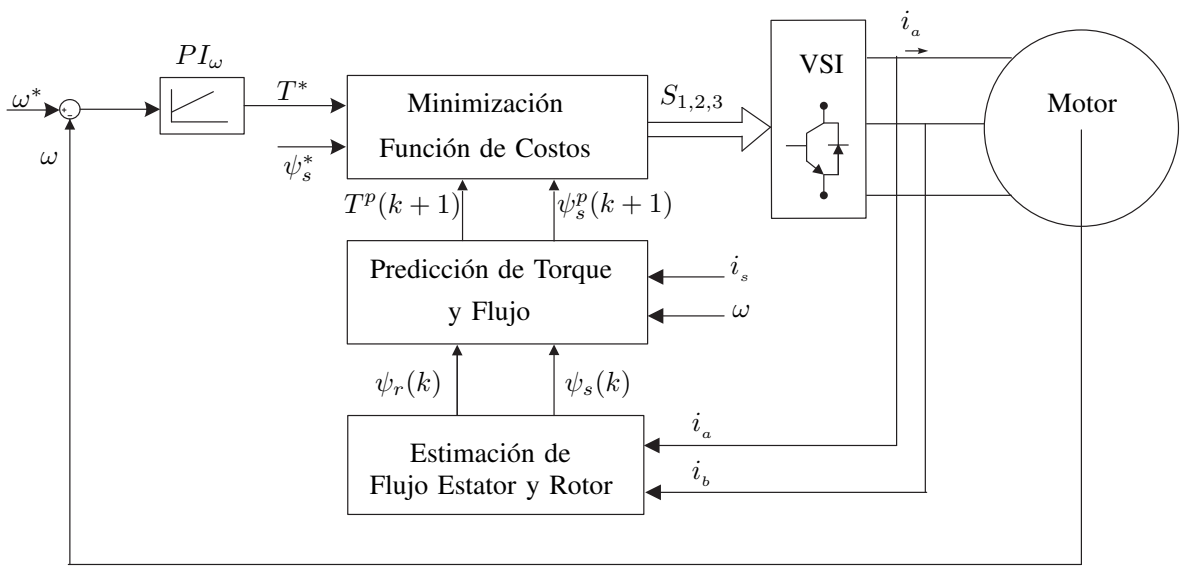

Fig. 2: Esquema de control para control predictivo de torque.

\section{TéCnica de Control Propuesto}

\section{A. Esquema de Control}

Para las máquinas de inducción, es posible demostrar que tanto el torque electromagnético $T_{e}$ como el flujo del estator pueden modificarse seleccionando una secuencia de vector de voltaje adecuada que modifique la magnitud del flujo del estator y al mismo tiempo aumente o disminuya el ángulo entre el rotor y el flujo del estator.

La técnica de control predictivo de torque (PTC), utiliza el mismo principio, pero en este esquema se calculan las predicciones para los valores futuros del flujo y torque del estator. Es por esto que la condición de referencia, que se implementa mediante una función de costo, toma en consideración el futuro comportamiento de estas variables. Las predicciones se calculan para cada posibilidad de activación y la función de costo selecciona el estado de conmutación (vector de voltaje) que optimiza el seguimiento de la referencia.

El esquema de control propuesto está indicado en la Fig. 2. El circuito de potencia considera el inversor fuente de voltaje y la máquina de inducción.

En el esquema de control, se distingue un controlador PI, el cual es el encargado de realizar el control de la velocidad del motor, generando así la referencia del torque eléctrico.

Las mediciones de corriente del motor son utilizadas para realizar la estimación de los flujos de rotor y estator, las cuales son a su vez utilizadas para, en conjunto con la medición de velocidad, predecir el torque y flujo de la máquina de inducción en el siguiente instante de muestreo. Estas predicciones de torque y flujo son obtenidas para cada uno de los 8 estados válidos del inversor fuente de voltaje.

Posteriormente, estas predicciones son comparadas con sus respectivas referencias en el bloque que minimiza la función de costos, seleccionando el estado de conmutación que genere el mínimo error entre las referencias y su respectiva predicción.
Finalmente, este estado de conmutación óptimo, es el seleccionado para ser aplicado el convertidor en el siguiente instance de tiempo.

\section{B. Modelo de Predicción}

El modelo del motor de inducción en la representación del vector espacial queda definido en (10) y (11), donde las ecuaciones de voltaje del estator y rotor en coordenadas fijas se presentan como:

$$
\begin{gathered}
\mathrm{v}_{\mathrm{s}}=R_{s} \mathrm{i}_{\mathrm{o}}+L_{s} \frac{d \psi_{s}}{d t} \\
\mathrm{v}_{\mathrm{r}}=R_{r} \mathrm{i}_{\mathrm{r}}+L_{r} \frac{d \psi_{r}}{d t}-j p \omega \psi_{s}
\end{gathered}
$$

$R_{s}, R_{r}$ y $\omega$ corresponden a la resistencia del estator, resistencia del rotor y la frecuencia angular del rotor. Los flujos del estator y rotor están relacionados con sus respectivas corrientes a través de las siguientes ecuaciones:

$$
\begin{aligned}
& \psi_{s}=L_{s} \mathrm{i}_{\mathrm{o}}+L_{m} \mathrm{i}_{\mathrm{r}} \\
& \psi_{r}=L_{r} \mathrm{i}_{\mathrm{r}}+L_{m} \mathrm{i}_{\mathrm{s}}
\end{aligned}
$$

Para obtener la estimación del flujo del estator y rotor, se discretizan las ecuaciones (10) y (11) utilizando la fórmula general de Euler con diferencia de avance utilizada como la aproximación derivada para estimar el valor de cada función en un tiempo de muestreo futuro.

La estimación del flujo del rotor y estator se definen en (14) y (15) :

$$
\begin{aligned}
& \psi_{s}(k)=\psi_{s}(k-1)+T_{s} \mathrm{v}_{o}(k)-R_{s} T_{s} \mathrm{i}_{o}(k) \\
& \psi_{r}=\frac{L_{r}}{L_{m}} \psi_{s}(k-1)+\left(L_{m}-\frac{L_{r} L_{s}}{L_{m}}\right) \mathrm{i}_{o}(k)
\end{aligned}
$$


Tabla II: Parámetros de Simulación del Control Predictivo de Torque de una Máquina de Inducción Alimentada por un VSI.

\begin{tabular}{c|c|c} 
Parámetros & Variables & Valor \\
\hline$T_{s}$ & Tiempo de muestreo & $50[\mu s]$ \\
$R_{s}$ & Resistencia de estator & $1.35[\Omega]$ \\
$R_{r}$ & Resistencia de rotor & $7.2[\Omega]$ \\
$L_{s}$ & Inductancia de magnetización estator & $0.282[\mathrm{H}]$ \\
$L_{r}$ & Inductancia de magnetización rotor & $0.282[\mathrm{H}]$ \\
$L_{m}$ & Inductancia mutua & $0.286[\mathrm{H}]$ \\
$J$ & Inercia & $0.062\left[\mathrm{Kgm}^{2}\right]$ \\
$p$ & Pares de polos & 2 \\
$p$ & Velocidad nomimal & $1430[\mathrm{rpm}]$ \\
$T_{n o m}$ & Torque nominal & $50[\mathrm{Nm}]$ \\
$T_{l}$ & Torque de carga & $40[\mathrm{Nm}]$ \\
$v_{d c}$ & Voltaje de alimentación & $520[\mathrm{~V}]$ \\
& Tiempo de simulación & $0.9[\mathrm{~s}]$
\end{tabular}

Una vez que se han obtenido las estimaciones del flujo del rotor y del estator, se deben calcular los valores futuros para las variables controladas. En el caso de control predictivo de torque, el torque electromagnético $T_{e} \mathrm{y}$ el flujo del estator se predicen para el siguiente instante de muestreo $(k+1)$. Para la predicción del flujo del estator $\psi_{s}^{p}(k+1)$, se toma en consideración la misma ecuación de voltaje del estator utilizada para su estimación [16] . Al aproximar la derivada del flujo del estator, se obtiene la predicción para $\psi_{s}^{p}$ :

$$
\psi_{s}^{p}(k+1)=\psi_{s}(k)+T_{s} \mathrm{v}_{o}(k+1)-R_{s} T_{s} \mathrm{i}_{o}(k+1)
$$

La predicción del torque queda definida como

$$
T_{e}=\frac{2}{3} p\left(\psi_{s} \times \mathrm{i}_{o}\right)
$$

Teniendo en cuenta las predicciones del flujo y corriente del estator, la predicción del torque queda determinada por

$$
T_{e}^{p}(k+1)=\frac{3}{2} p\left[\psi_{s}^{p}(k+1) \times \mathrm{i}_{o}^{p}(k+1)\right]
$$

De acuerdo a la ecuación para la predicción del torque (18), se requiere obtener una predicción de la corriente del estator $\mathrm{i}_{o}^{p}(k+1)$, para calcular una predicción del torque electromagnético. Es por esto que se debe discretizar (8) y reemplazar las derivadas obtenidas por la aproximación basada en Euler [15], obteniendo la estimación de la corriente de estator $\mathrm{i}_{o}$ para el instante $(k+1)$ :

$$
\begin{aligned}
& \mathrm{i}_{o}^{p}(k+1)=\left(1-\frac{T_{s} r_{\sigma}}{L_{s} \sigma}\right) \mathrm{i}_{o}(k)+ \\
& \frac{T_{s}}{L_{s} \sigma}\left[\left(\frac{k_{r}}{\tau_{r}}-j k_{r} \omega(k)\right) \psi_{\mathrm{r}}(\mathrm{k})+\mathrm{v}_{\mathrm{o}}(\mathrm{k}+1)\right]
\end{aligned}
$$

Una vez obtenidas las predicciones de flujo de corriente del estator, es posible calcular la predicción del torque electromagnético. Tanto la predicción del torque y flujo del estator se escriben en términos del voltaje del inversor $\mathrm{v}_{s}(k)$. Esto implica que se obtienen siete predicciones diferentes para el torque y el flujo, de acuerdo con el número de estados de conmutación válidos (vectores de voltaje) generados por un inversor de dos niveles.

\section{Función de Costo}

La selección del estado óptimo de conmutación a ser aplicado se debe obtener minimizando una función de costo, la cual corresponde al cálculo del error entre las referencias de torque y flujo y sus respectivos valores predichos. La función de costo se evalúa para cada estado de conmutación y se selecciona aquél que produce el error más bajo. De este modo, se generan los pulsos de disparo del inversor.

La función de costos se define como:

$g=\left|T_{e}^{*}-T_{e}^{p}(K+1)\right|+\lambda_{T}\left|\psi_{s}^{*}-\psi_{s}^{p}(k+1)\right|$

Donde $T_{e}^{*}$ y $\psi_{s}^{*}$ corresponden a los torque y flujo de referencia. $\lambda_{T}$, corresponde al factor de peso, este factor aumenta o disminuye la importancia relativa del torque en comparación con el control de flujo y es la razón entre los valores nominales de torque $T_{n o m}$ y el flujo del estator [17]:

$$
\lambda_{T}=\frac{T_{n o m}}{\psi_{s}}
$$

\section{Resultados}

Con el fin de validar la implementación del esquema de control propuesto, éste se simuló en Matlab/Simulink, considerando los parámetros indicados en la Tabla II.

Tal como se observa en la Fig. 3, es posible obtener un buen seguimiendo de la velocidad mecánica del motor, demostrando el buen funcionamiento del controlador PI (Fig. 3a). La velocidad de referencia alcanza la velocidad nominal del rotor de $1430[\mathrm{rpm}]$ en el instante de tiempo $t=0.02$ $[s]$. Cuando el motor comienza a acelerar, logra alcanzar la velocidad nominal a los $0.18[s]$ aproximadamente.

Este controlador PI genera la referencia para el torque eléctrico, cuyo seguimiendo se observa en la Fig. 3b. Aquí se aprecia un torque eléctrico con una rápida respuesta dinámica a su respectiva referencia. Mientras el motor está acelerando hasta el tiempo $\mathrm{t}=0.18[s]$, el torque medido se estabiliza siguiendo correctamente el torque de referencia. En el tiempo $\mathrm{t}=0.3[\mathrm{~s}]$ mientras el motor ya está girando a su velocidad nominal, se aplica un torque de carga, presentando una rápida respuesta ante este escalón siguiendo rápidamente el torque de 

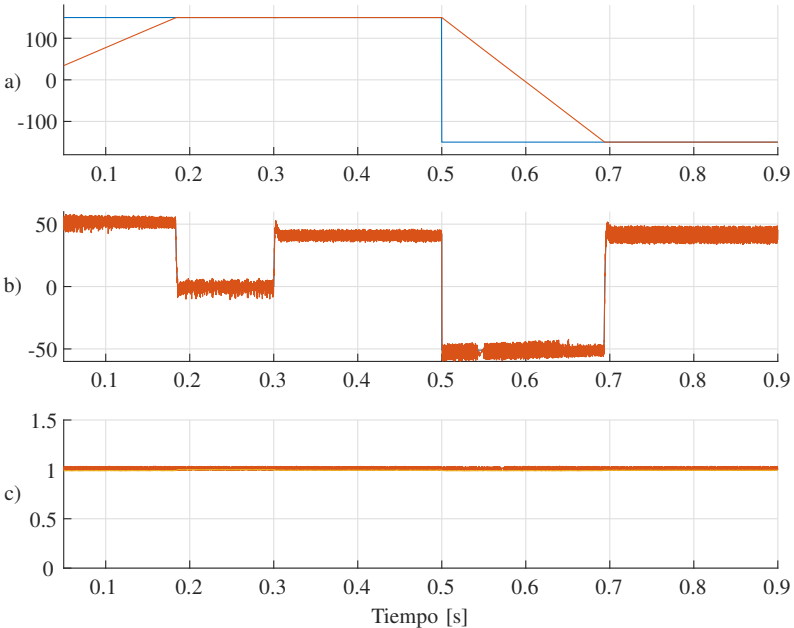

Fig. 3: Resultados de simulación del esquema de control predictivo de torque y flujo: a) referencia de velocidad y su valor medido [rad/s], b) referencia de torque y su valor medido $[\mathrm{Nm}]$, c) flujo de referencia y estimado del estator [Wb].
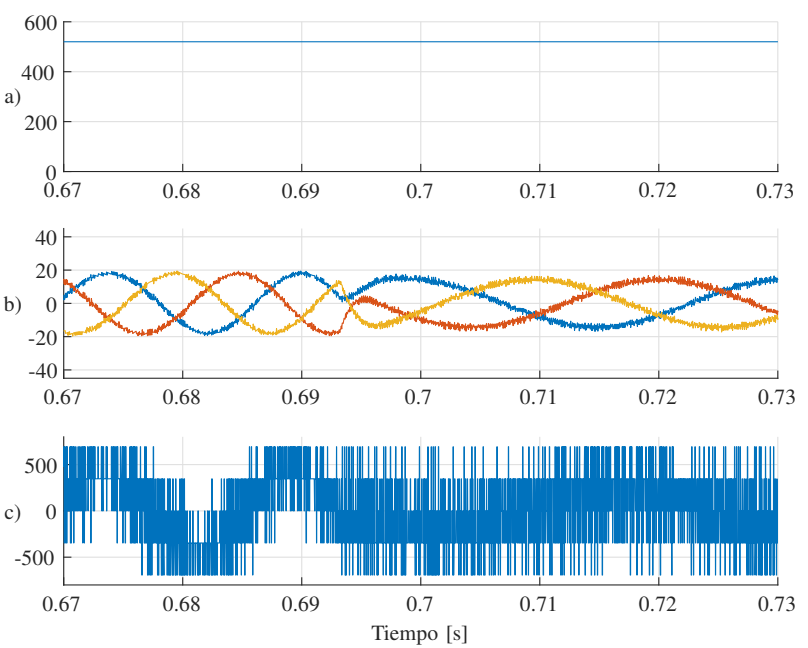

Fig. 4: Resultados de simulación del esquema de control predictivo de torque y flujo: a) voltaje del enlace DC $v_{d c}[\mathrm{~V}]$, b) corrientes en la salida del VSI $\left.\mathbf{i}_{\mathbf{o}}[\mathrm{A}], \mathrm{c}\right)$ voltaje de salida del convertidor $v_{a n}[\mathrm{~V}]$.

referencia y manteniéndose después de la inversión de marcha ocurrida en el instante $\mathrm{t}=0.5[\mathrm{~s}]$. Igualmente, el flujo de estator sigue a su referencia tal como se indica en la Fig. 3c.

Respecto a las variables del inversor fuente de voltaje, es posible observar en la Fig. 4, para una escala reducida de tiempo [s], el voltaje en el DC-link $v_{d c}$ el cual está establecido en $v_{d c}=520[\mathrm{~V}]$, las corrientes de carga $\mathbf{i}_{\mathbf{o}}$ en coordenadas $\alpha$ $\beta$ [A] y el voltaje de carga $v_{a n}$ el cual presenta una forma de onda PWM variable, dependiendo del punto de operación.

\section{CONCLUSIONES}

En este paper se ha detallado la implementación de un control predictivo de torque y flujo de un motor de inducción alimentado por un inversor fuente de voltaje. El esquema es simple de implementar, demostrando muy buen desempeño al seguimiento de velocidad, torque y flujo, considerándose una alternativa a las técnicas clásicas de control.

\section{AGRADECIMIENTOS}

Los autores agradecen al financiamiento del Programa FONDECYT Regular a través del proyecto 1191028, MEC 80190074, MEC 801800974 y FONDAP SERC Chile 15110019 .

\section{REFERENCIA}

[1] X. Zhang and G. H. B. Foo, "Overmodulation of constant-switchingfrequency-based DTC for reluctance synchronous motors incorporating field-weakening operation," IEEE Trans. Ind. Electron., vol. 66, no. 1, pp. 37-47, Jan 2019.

[2] K. M. R. Eswar, K. V. P. Kumar, and T. V. Kumar, "A simplified predictive torque control scheme for open-end winding induction motor drive," IEEE J. of Emerg. Sel. Topics in Power Electron., vol. 7, no. 2, pp. 1162-1172, June 2019.

[3] F.Blaschke, "The principle of field orientation as applied to the new transvector closed loop control for rotating field machines," Siemens Rev, pp. 217-220, 1972.

[4] B. Wu and M. Narimani, High-Powe Converters and AC drives. John Wiley, 2017, vol. 2

[5] P. Karamanakos and T. Geyer, "Model predictive torque and flux control minimizing current distortions," IEEE Trans. Power Electron., vol. 34 no. 3, pp. 2007-2012, March 2019.

[6] F. Wang, H. Xie, Q. Chen, S. A. Davari, J. Rodrguez, and R. Kennel, "Parallel predictive torque control for induction machines without weighting factors," IEEE Transactions on Power Electronics, vol. 35, no. 2, pp. 1779-1788, 2020.

[7] G. S. Buja and M. P. Kazmierkowski, "Direct torque control of pwm inverter-fed ac motors - a survey," IEEE Transactions on Industrial Electronics, vol. 51, no. 4, pp. 744-757, 2004.

[8] F. Niu, B. Wang, A. S. Babel, K. Li, and E. G. Strangas, "Comparative evaluation of direct torque control strategies for permanent magnet synchronous machines," IEEE Transactions on Power Electronics, vol. 31, no. 2, pp. 1408-1424, 2016.

[9] A. Salem, M. Mamdouh, and M. A. Abido, "Predictive torque control and capacitor balancing of a sic-based dual t-type drive system," IEEE Transactions on Power Electronics, vol. 35, no. 3, pp. 2871-2881, 2020.

[10] J. Rodriguez and P. Cortes, Predictive control of power converters and electrical drives. John Wiley \& Sons, 2012, vol. 40.

[11] H. Miranda, P. Cortes, J. I. Yuz, and J. Rodriguez, "Predictive torque control of induction machines based on state-space models," IEEE Transactions on Industrial Electronics, vol. 56, no. 6, pp. 1916-1924, 2009.

[12] P. Guazzelli, W. de Andrade Pereira, C. de Oliveira, A. de Castro, and M. de Aguiar, "Weighting factors optimization of predictive torque control of induction motor by multiobjective genetic algorithm," IEEE Transactions on Power Electronics, vol. 34, no. 7, pp. 6628-6638, July 2019.

[13] F. Wang, Z. Zhang, S. A. Davari, R. Fotouhi, D. Arab Khaburi, J. Rodriguez, and R. Kennel, "An encoderless predictive torque control for an induction machine with a revised prediction model and EFOSMO," IEEE Transactions on Industrial Electronics, vol. 61, no. 12, pp. 66356644, Dec 2014.

[14] J. A. Riveros, M. Rivera, C. Rodrguez, M. Galea, G. Buticchi, and P. Wheeler, "Predictive torque control with fixed switching frequency for induction motor drives," in 2020 IEEE International Conference on Industrial Technology (ICIT), 2020, pp. 211-216.

[15] Predictive control of power converters and electrical drives. John Wiley \& Sons, 2012, ch. Predictive Control of Induction Machines.

[16] R. Vargas, U. Ammann, B. Hudoffsky, J. Rodriguez, and P. Wheeler, "Predictive torque control of an induction machine fed by a matrix converter with reactive input power control," IEEE Transactions on Power Electronics, vol. 25, no. 6, pp. 1426-1438, 2010.

[17] P. Cortes, S. Kouro, B. La Rocca, R. Vargas, J. Rodriguez, J. I. Leon, S. Vazquez, and L. G. Franquelo, "Guidelines for weighting factors design in model predictive control of power converters and drives," in 2009 IEEE International Conference on Industrial Technology, 2009, pp. $1-7$ 